\title{
Risk of Cardiovascular Death in the Remote Period after Myocardial Revascularization and in Association with Renal Dysfunction
}

\author{
Ekaterina S. Levitskaya, $\mathrm{PhD}^{1 *}$; Mikhail M. Batiushin, $\mathrm{PhD}, \mathrm{ScD}^{1}$; Aleksey V. Hripun, $\mathrm{PhD}^{1}$; \\ Aleksandr A. Kastanajan, $\mathrm{PhD}, \mathrm{ScD}^{1}$; Elena O. Golovinova ${ }^{1}$; Valentina V. Gul'chenko'; \\ Dmitriy G. Pasechnik, $\mathrm{PhD}^{1}$; Vladimir A. Chistyakov, $\mathrm{PhD}, \mathrm{ScD}^{1,2}$; \\ Igor V. Dudarev, $\mathrm{PhD}, \mathrm{ScD}^{1}$; Galina V. Shavkuta, $\mathrm{PhD}, \mathrm{ScD}^{1}$ \\ ${ }^{1}$ Rostov State Medical University, ${ }^{2}$ Southern Federal University \\ Rostov-on-Don, Russia
}

\begin{abstract}
The aim of the present study was to assess the effectiveness of standard medical therapy in lowering the risk of cardiovascular death in the remote period after myocardial revascularization (MR), taking into account the presence of renal dysfunction.

Material and Methods: The study included 90 patients with coronary heart disease (CHD) and indications for revascularization. We evaluated a drug therapy obtained at different stages of revascularization, as well as the severity of patients' condition and the prevalence of renal dysfunction.

Results: In the remote period after MR (5.8 \pm 0.05 years), $71 / 78.9 \%$ patients participated in the study; death occurred in $10 / 12.3 \%$ patients. The duration of therapy for chronic myocardial ischemia before MR $(P=0.005)$, as well as compliance with prescribed therapy during 6 months $(P=0.008)$ after this procedure, affected cardiovascular death in the remote period after MR. Using statins before MR reduced the risk of cardiovascular death by $17.2 \%(P=0.01)$, beta-blockers $-14.95 \%(P=0.04)$, and ACE inhibitors (ACEIs) - 15.75\% ( $P=0.03)$. The lack of regular use of acetylsalicylic acid (ASA) for 6 months after RM was associated with an increase in the risk of cardiovascular death up to $36.2 \%(P=0.005)$. Statins and ACEIs are drugs that reduce the risk of cardiovascular death in the presence of renal dysfunction $(P<0.05)$.

Conclusion: An efficient drug regimen for patients after MR is important in reducing a long-term prognosis of cardiovascular death and for an efficient correction of coronary artery patency. (Int J Biomed. 2016;6(1):12-17.).
\end{abstract}

Keywords: risk of cardiovascular death; ischemic heart disease; revascularization; renal dysfunction.

Abbreviations: LVEDV, left ventricular end-diastolic volume; LVESV, left ventricular end-systolic volume; IVST, interventricular septal thickness; LVPWT, left ventricular posterior wall thickness; LVMI, left ventricular mass index; LVEF, left ventricular ejection fraction; GFR, glomerular filtration rate; MAU, microalbuminuria.

\section{Introduction}

The effectiveness of various methods of treatment is one of the relevant aspects of research activity despite the development of modern medicine. The principle of evidence is the fundamental basis for the rational use and effectiveness of a therapy. Drugs with a broad evidence base are the means of first choice in the treatment of patients with any pathology. The right choice of drugs becomes a priority, especially in

*Corresponding author: Ekaterina S. Levitskaya, PhD. Rostov State Medical University, Rostov-on-Don, Russia. E-mail: es.med@mail.ru the treatment of socially significant diseases with a high risk of complications and death. Worldwide statistics indicate a primary role of the pathology of the cardiovascular system in the general structure of permanent disability and mortality from chronic non-communicable diseases [1,2], among which coronary heart disease (CHD) holds the leading position [3]. Patients with indications for surgical revascularization are the most disadvantaged group of patients regarding prognosis for cardiovascular death (CVD) and complications. Operations to restore coronary blood flow significantly improve the quality of life of patients and reduce the risk of developing acute myocardial infarction (AMI) and sudden CVD. The right selection of components of medical treatment for a 
group of patients after MR is justified by the necessity to slow the progression of the pathogenic mechanisms of the atherosclerotic process, and, as a consequence, to significantly increase the effectiveness of interventional treatment.

The presence of cardiovascular disease is often accompanied by damage to target organs or the formation of clinical conditions, the development of which is caused by a single pathogenesis. Developing multimorbidity potentiates a poor prognosis with cardiovascular complications and requires the use of the most rational drug combinations, which minimize the risk of the progression of disease, death, and acute vascular catastrophes.

The existing evidence base for drug therapy in patients after restoration of coronary blood flow includes statins and low doses of acetylsalicylic acid (ASA), as well as ACE inhibitors (ACEIs) and beta-blockers in certain clinical situations [4]. Research and clinical activities seek to obtain information that complements the known data regarding the priority drug regimens. It is important to emphasize the special significance and the practical value of this information in the evaluation of long-term prognosis of cardiovascular events.

The aim of the present study was to assess the effectiveness of standard medical therapy in lowering the risk of cardiovascular death (rCVD) in the remote period after myocardial revascularization (MR), taking into account the presence of renal dysfunction (RD).

\section{Materials and Methods}

The present study included 90 CHD patients (80 men and 10 women; mean age $56.1 \pm 0.9$ years) with indications for restoration of coronary blood flow. The study was conducted in accordance with ethical principles of the Declaration of Helsinki and approved by the Rostov State Medical University Ethics Committee. Written informed consent was obtained from all participants.

The inclusion criteria were indications for surgical revascularization of the myocardium by the method of coronary artery bypass grafting $(\mathrm{CABG})$ or percutaneous coronary intervention (PCI) with stent implantation. The need to restore coronary blood flow was determined by the results of coronary angiography (CAG). After completing the patient recruitment in the study group, we found that $\mathrm{CABG}$ was required in $64 / 57.6 \%$ patients and PCI with stent implantation in $26 / 42.4 \%$ patients.

At the beginning of the study, we assessed the prevalence of traditional risk factors (RFs): the presence and duration of smoking, arterial hypertension $(\mathrm{AH})$, overweight and obesity, diabetes mellitus (DM), and the presence a prior MI and duration of CHD. We then estimated the biochemical markers (blood lipids and type of dyslipidemia), the main echocardiographic parameters (LVEDV, LVESV, IVST, LVPWT, LVMI, and LVEF), and the parameters of renal function - MAU and GFR. MAU (urinary albumin excretion of $30-300 \mathrm{mg} / 24$ hours) was assessed by a semiquantitative method using test strips for the determination of protein in the urine, in compliance with the rules for collecting morning urine. GFR was estimated by the Cockcroft-Gault formula. Stages of chronic kidney disease (CKD) were determined according to the KDOQI 2002 classification. Patients with CKD stages 4-5 were excluded from this study.

Clinical characteristics of the studied groups are presented in Table 1.

Table 1.

Cardiovascular risk factors and clinical characteristics of CHD patients

\begin{tabular}{|l|c|}
\hline \multicolumn{1}{|c|}{ Criterion } & Average value \\
\hline Duration of therapy of CHD, years & $6.1 \pm 0.6$ \\
\hline Patients with excess body weight, abs (\%) & $34(37.8)$ \\
\hline Obesity, abs (\%) & $43(47.8)$ \\
\hline Smoking patients, abs (\%) & $32(35.6)$ \\
\hline Total cholesterol, mmol/1 & $5.65 \pm 0.15$ \\
\hline High-density lipoprotein cholesterol, mmol/1 & $1.1 \pm 0.03$ \\
\hline Low-density lipoprotein cholesterol, mmol/1 & $4.6 \pm 0.2$ \\
\hline Triglycerides, mmol/1 & $1.95 \pm 0.1$ \\
\hline Dyslipidemia, type IIa, abs (\%) & $47(52.2)$ \\
\hline Dyslipidemia, type IIb, abs (\%) & $29(32.2)$ \\
\hline LVEDV, ml & $151.2 \pm 3.9$ \\
\hline LVESV, ml & $72.3 \pm 3.01$ \\
\hline IVST, mm & $12.53 \pm 0.17$ \\
\hline LVPWT, mm & $11.99 \pm 0.15$ \\
\hline LVEF, \% & $53.0 \pm 0.7$ \\
\hline Angina pectoris, class II, abs (\%) & $5(5.6)$ \\
\hline Angina pectoris class III, abs (\%) & $73(81.1)$ \\
\hline Angina pectoris, class IV, abs (\%) & $1(1.1)$ \\
\hline Unstable angina, abs (\%) & $8(8.9)$ \\
\hline Acute myocardial infarction, abs (\%) & $3(3.3)$ \\
\hline Myocardial infarction, abs (\%) & $66(73.3)$ \\
\hline Arterial hypertension, abs (\%) & $77(85.6)$ \\
\hline Diabetes, abs (\%) & $19(21.1)$ \\
\hline MAU, abs (\%) & $92.2 \pm 2.2$ \\
\hline GFR, mL/min & $33(53.3)$ \\
\hline CKD stage 1, abs (\%) & $7(7.8)$ \\
\hline CKD stage 2, abs (\%) & \\
\hline CKD stage 3, abs (\%) & (\%) \\
\hline
\end{tabular}

Statistical analysis of the obtained data allowed us to establish the high prevalence of both traditional and renal risk factors in the studied group of patients. To achieve the study objective, we assessed CHD therapy duration before the revascularization, as well as groups of drugs, receipt of which was regular. The average duration of CHD medical treatment was $1.57 \pm 0.27$ years.

Medical therapy aimed at slowing the progression of the atherosclerotic process was recommended to all patients after MR. We performed a statistical analysis of regular use of medicines also in the late period, $6.3 \pm 0.04$ months after MR. Analysis of the frequency of regular medication intake before MR and in the late period after the intervention is presented in Table 2. On the second visit in the late period after coronary reperfusion, we also monitored the presence of MAU and GFR value.

The endpoint of the study was CVD in the remote period after MR (5.8 \pm 0.05 years). CVD occurred in $10 / 12.3 \%$ of 81 patients who continued participation in the study. 
Table 2.

The frequency of regular medication intake (abs/\%) before MR and in the late period after the intervention

\begin{tabular}{|l|c|c|}
\hline \multicolumn{1}{|c|}{ Medications } & Before MR & After MR $(\approx 6.3 \mathrm{mth})$ \\
\hline Statins & $40 / 44.4$ & $74(84.1)$ \\
\hline ASA & $53 / 58.9$ & $79(89.8)$ \\
\hline Beta-blockers & $55 / 61.1$ & $73(83.0)$ \\
\hline ACE inhibitors & $48 / 53.3$ & $69(78.4)$ \\
\hline Calcium channel blockers & $7 / 7.8$ & $10(11.4)$ \\
\hline Diuretics & $5 / 5.6$ & $12(13.3)$ \\
\hline Sartans & $2 / 2.2$ & $2(2.2)$ \\
\hline Nitrates & $33 / 36.7$ & $18(20.0)$ \\
\hline Trimetazidine & $15 / 16.7$ & $19(21.1)$ \\
\hline
\end{tabular}

Statistical analysis of data was performed using the software Statistica 8.0. The mean (M) and standard error of mean (SEM) were calculated. Differences of continuous variables with a normal distribution between the two groups were calculated using the independent-sample $t$-test. Group comparisons with respect to categorical variables are performed using chi-square tests. Two-tailed $P$ values $<0.05$ were considered statistically significant.

\section{Results}

rCVD in the remote period after MR decreased significantly with increased duration of regular CHD drug therapy prior to MR. Thus, regular treatment for 4 years was associated with a low risk of death amounting to $0.09 \%$, but the absence of drug therapy led to an increased risk of death in the remote period after MR by $23.8 \%(P=0.005)$ (Figure 1$)$.

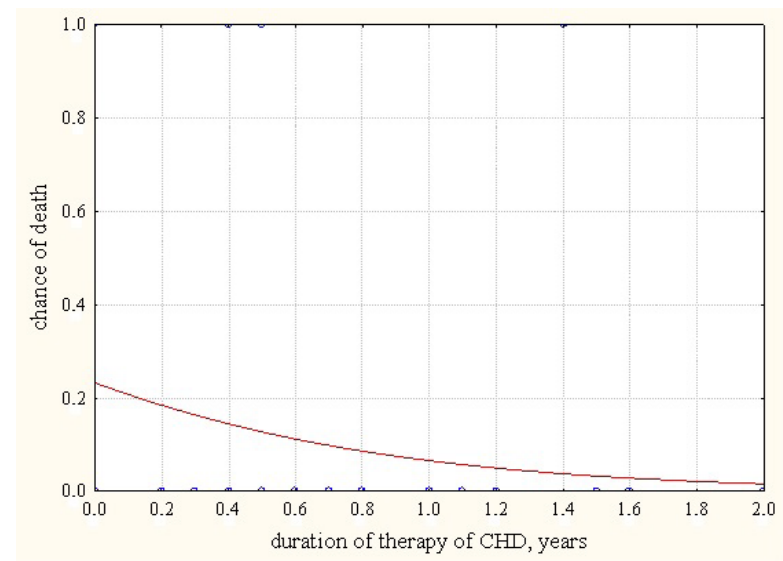

Fig. 1. The probability of death in remote period after $M R$ depending on the duration of CHD therapy

Statistical data obtained when analyzing the frequency of use of drug classes before MR showed that statins, betablockers, and ACEIs significantly reduced rCVD. It should be noted that the degree of influence on cardiovascular prognosis was comparable for the presented classes. rCVD in the remote period after MR was $20.0 \%$ in the absence of continuous use of statins in the period before MR $(P=0.01)$; this rate was $21.2 \%$ for beta-blockers $(\mathrm{P}=0.04)$ and $20.51 \%$ for ACEIs $(P=$ $0.03)$. Comparison of patient groups with the presence and absence of taking statins, beta-blockers and ACEIs before coronary reperfusion has allowed us to establish an increase in the probability of death among patients who did not take these drugs by $17.22 \%, 14.95 \%$ and $15.75 \%$, respectively.

In patients with continuous ASA use before MR, the impact on rCVD reduction in the remote period after MR approached statistically significant values. The results of the regular medication intake in the later period ( $6.3 \pm 0.04$ months) after MR showed that continuous use of the recommended regimens leads to significant $\mathrm{rCVD}$ reduction in the remote period after MR by $40.8 \%(P=0.008)$.

We have shown the high importance of ASA use in reducing $\mathrm{rCVD}$ by $36.2 \%(P=0.009)$. after $5.8 \pm 0.05$ years following MR. When we analyzed the impact of ACEIs, betablockers and statins on rCVD in the remote period after MR, reliable results had not been established; at the same time, we identified a trend of statistical significance in the regular use of beta-blockers.

In the presence of MAU determined before revascularization, regular ACEI intake was accompanied by reduction of $\mathrm{rCVD}$ in the later period after MR by $10.22 \%$ $(P=0.03)$, and statins by $5.67 \%(P=0.02)$.

In the analysis of GRF value calculated before MR, we found that in patients with normal filtration capacity of the kidneys, or a tendency to hyperfiltration combined with regular intake of ACEIs $(P=0.04)$ or statins $(P=0.03)$, rCVD was lower in comparison to patients with decreased GRF (Figures 2 and 3).

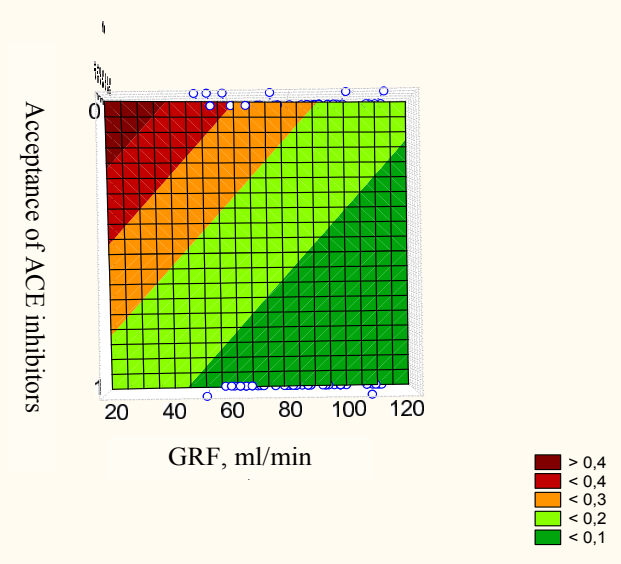

Fig. 2. The probability of death in remote period after MR depending on the regular ACEIs intake and GFR level

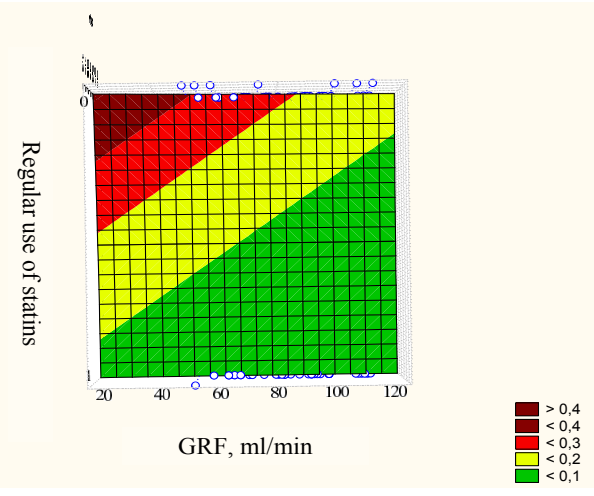

Fig. 3. The probability of death in remote period after MR depending on the regular statin intake and GFR level 
We revealed $\mathrm{rCVD}$ reduction during regular intake of the prescribed regimen for 6 months after MR in patients with MAU and decreased GFR values. Continuous use of drugs for CHD treatment was associated with a decrease in $\mathrm{rCVD}$ by $11.9 \%$ in patients with MAU $(P=0.02)$. We found a more significant decrease in the probability of death during medical therapy in patients with more impaired kidney filtration function. ASA intake was associated with rCVD reduction by $36.9 \%$ in patients with MAU $(P=0.03)$. The risk of death amounted to $42.1 \%$ at GFR of $90 \mathrm{ml} / \mathrm{min}$ and ASA absence in the drug regime, while a regular ASA admission within 6 months after MR decreased the probability of death by $7.7 \%$; these risks amounted to $64.1 \%$ and $17.7 \%(P=0.02)$, respectively in a GFR value of $30 \mathrm{ml} / \mathrm{min}$.

\section{Discussion}

Evidence-based drug therapy is the basis for cardiovascular risk reduction. Surgical MR is the most effective method to treat coronary insufficiency that improves the quality of life of patients and predicts cardiovascular complications. At the same time, surgical restoration of intracoronary blood flow is not a tool that controls CVD pathogenesis. The further tactics for management of patients after MR should include a choice of the optimal medical treatment, which can effectively delay mechanisms of restenosis formation and reduce the risk of cardiovascular complications.

In the present study, we showed that the remote rCVD in patients receiving long-term anti-ischemic therapy was significantly lower in comparison to patients with short-term periods of drug therapy or the lack of it. It should be noted that the probability of death becomes minimal with an increase in the duration of therapy, whereas a fatal outcome can be predicted in almost one of four patient in the absence of the regular therapy.

Numerous studies have shown that statins, ACEIs and beta-blockers may reduce rCVD. Moreover, a significant decrease in $\mathrm{rCVD}$ in the remote period after MR was obtained under the condition of regular reception of these drug classes before surgical correction of coronary blood flow. Comparative analysis of the drug classes used within 6 months after coronary reperfusion has allowed us to establish the primary importance of ASA in reducing the risk of a fatal outcome in the long term. Based on these data, one can judge the priority of correction of the pathogenetic mechanisms of CHD progression depending on the treatment stage. Reducing the activity of neurohumoral systems, improving the endothelial function, and normalizing the lipid spectrum are the most important treatments in the period before MR, while an effective control of platelet activity is the priority in the near term after MR. Our results are consistent with numerous studies devoted to drug therapy in CHD patients.

Philip F. et al. [5] conducted a retrospective cohort study of 5,205 patients after first-time isolated CABG and assessed the impact of a discharge regimen including beta-blockers and statin therapy and their relationship to long-term all cause mortality and major adverse cardiovascular events. A discharge regimen with statin therapy was associated with and overall reduction in 30 day, 1 year and long-term mortality. In addition, statin and beta-blockers exerted synergistic effect on overall mortality outcomes short-term and in the long-term.

There are many studies confirming the protective role of statins in patients after MR, in which the probability of occlusion and rCVD was decreased by reducing the atherosclerotic process progression in a general coronary bed and arteries subjected to surgical correction [6,7].

The necessity of using beta-blockers in CHD patients is not in doubt and is dictated by the peculiarity of the mechanism of their action. Recent studies of beta-blockers used in patients after CABG demonstrate the need to include this drug class for continuous use.

The study conducted by Zhang $\mathrm{H}$ et al. [8] included 5926 consecutive patients who underwent CABG and were discharged alive. The prevalence and consistency of $\beta$-blocker use were determined in patients with and without a history of myocardial infarction (MI). In patients with or without previous $\mathrm{MI}$ undergoing $\mathrm{CABG}$, the consistent use of $\beta$-blockers was associated with a lower risk of long-term mortality and adverse cardiovascular events.

Our earlier studies showed a need of beta-blocker use after MR for correction of a recurrent angina, which is a marker of coronary atherosclerosis progression and adverse cardiovascular events [9]. In the group of patients after 6 months following MR, we found that beta-blocker use before coronary reperfusion reduced the risk of recurrence of angina by $33.0 \%$ and a dose-dependent effect was demonstrated [9].

Suppression of the influence of angiotensin II by angiotensin-converting enzyme inhibitors and angiotensin II receptor blockers may reduce or potentially reverse atherosclerosis and other inflammation-associated cardiovascular diseases [10].

L.Minuzzo et al [11] investigated the effect of previous use of angiotensin-converting enzyme inhibitors on cardiac troponin I measurement in patients with acute coronary syndrome without ST-segment elevation and clinical outcomes at 180 days. This study showed a correlation between prior use of ACEIs and reduction in the myocardial necrosis marker troponin I in patients admitted for acute coronary syndrome without ST-segment elevation. However, large-scale trials are still required to state that this reduction could lead to fewer severe clinical events such as death and re-infarction.

Current guidelines consider the validity of ACEI assignment for certain clinical situations in chronic CHD [4]. It is important to note that patients included in our study were characterized by a high prevalence of impaired kidney function. In this regard, the results obtained can be justified in view of the correction of cardio-renal syndrome with a significantly improved long-term cardiovascular prognosis.

The importance of optimal drug combinations increases significantly in the presence of multimorbidity, especially with the existence of conditions united by a common pathogenesis and risk factors. Development of secondary nephropathy of cardiac origin increases a poor prognosis and requires the most effective approaches in the pharmacological treatment. RD markers such as MAU and a decreased GFR are the most significant indicators in the formation of an 
adverse cardiovascular prognosis. The strategy of medical management of such patients should be based on the concept of cardio-nephroprotection.

We have shown that a significant decrease in rCVD in patients with MAU/decreased GFR is associated with a regular application of ACEIs and statins prior to revascularization. Regular intake of these drugs allows not only reduction of $\mathrm{rCVD}$, but also equalizes the magnitude of this risk in the presence and absence of RD. At the same time, the tendency towards hyperfiltration reduces the risk even in the absence of a permanent use of ACEIs or statins. The obtained information about the role of statins in cardiovascular complication risk correction in RD presence is consistent with results of other studies. According to R. Agarwal [12], subgroup analyses of major clinical studies and meta-analyses of smaller trials indicate that statin therapy slows the decline of the glomerular filtration rate. Additionally, statins appear to reduce proteinuria in patients with CKD. Statins are well recognized to reduce cardiovascular morbidity and mortality in patients with and without documented cardiovascular disease and in certain high-risk populations, such as persons with diabetes mellitus. Statins have also been shown to preserve glomerular filtration rate and reduce proteinuria in subjects with nondiabetic renal disease. However, conclusive evidence for improved cardiovascular outcomes with statin therapy for CKD is not yet available. Thus, atorvastatin had no statistically significant effect on the composite primary end point of cardiovascular death, nonfatal myocardial infarction, and stroke in patients with diabetes receiving hemodialysis [13]. According to B.Fellström et al. [14], in patients undergoing hemodialysis, the initiation of treatment with rosuvastatin lowered the LDL cholesterol level but had no significant effect on the composite primary end point of death from cardiovascular causes, nonfatal myocardial infarction, or nonfatal stroke. At the same time, the SHARP trial showed that reduction of LDL cholesterol with simvastatin $20 \mathrm{mg}$ plus ezetimibe $10 \mathrm{mg}$ daily safely reduced the incidence of major atherosclerotic events (non-fatal myocardial infarction or coronary death, non-haemorrhagic stroke, or any arterial revascularisation procedure) in a wide range of patients with advanced chronic kidney disease [15].

Analysis of data in the literature and our results can be explained by the fact that the reduction of rCVD with statin therapy is slight in the presence of RD, whereas normal renal function or a tendency towards hyperfiltration has a protective effect. Long-term use of statins [12] and ACEIs [16,17] helps to slow down kidney remodeling, leading thereby to reduction of the progression of $\mathrm{RD}$ and $\mathrm{rCVD}$.

\section{Conclusion}

The assignment of rational treatment regimens and RF management for patients at very high risk of cardiovascular complications is essential to maximum prognosis improvement of these patients. Patients after MR need an effective slowdown of the pathogenic mechanisms of disease progression with the definition of schemes and algorithms for drug therapy taking into account variations in clinical situations. The results of our study allow us to highlight the main points for the effective improvement of cardiovascular prognosis and $\mathrm{rCVD}$ reduction in the remote period after MR:

- A long-term treatment with drugs correcting processes of chronic ischemia is strongly recommended prior to planned surgical MR.

- Statins, beta-blockers, and ACEIs are most effective before MR.

- The use of ASA for at least 6 months in the postoperative period significantly improves the prognosis.

- Adherence to the prescribed CHD therapy after MR is an important condition for a favorable prognosis.

- The inclusion in the therapeutic regimens of statins and ACEIs before and ASC after MR is recommended in the presence of MAU and decreased GFR.

- An assessment of renal function is important for rCVD determination.

\section{Competing interests}

The authors declare that they have no competing interests.

\section{Acknowledgment}

V. Chistyakov was supported by grant $6.1202 .2014 / \mathrm{K}$ from the Ministry of Education and Science of the Russian Federation.

\section{References}

1. World Health Organization. Global Status Report on Noncommunicable Diseases 2014. http://apps.who.int/iris/ bitstream/10665/148114/1/9789241564854_eng.pdf

2. Castellano JM, Narula J, Castillo J, Fuster V. Promoting Cardiovascular Health Worldwide: Strategies, Challenges, and Opportunities. Rev Esp Cardiol (Engl Ed). 2014; 67(9): 724-30.

3. Tuso P, Stoll SR, Li WW. A Plant-based diet, atherogenesis, and coronary artery disease prevention. Perm J. $2015 ; 19(1): 62-7$.

4. 2014 ESC/EACTS Guidelines on myocardial revascularization: The Task Force on Myocardial Revascularization of the European Society of Cardiology (ESC) and the European Association for Cardio-Thoracic Surgery (EACTS)Developed with the special contribution of the European Association of Percutaneous Cardiovascular Interventions (EAPCI). Authors/Task Force members, Windecker S, Kolh P, Alfonso F, Collet JP, Cremer J, Falk V, et al. Eur Heart J. 2014 Oct 1;35(37):2541-619.5.

5. Philip F, Blackstone E, Kapadia SR. Impact of statins and beta-blocker therapy on mortality after coronary artery bypass graft surgery. Cardiovasc Diagn Ther. 2015; 5(1): 8-16. 6. LaRosa JC, Grundy SM, Waters DD, Shear C, Barter P, Fruchart JC, et al. Intensive lipid lowering with atorvastatin in patients with stable coronary disease. N Engl J Med. 2005; 352(14):1425-35.

7. Kulik A, Brookhart MA, Levin R, Ruel M, Solomon DH, Choudhry NK. Impact of statin use on outcomes after coronary artery bypass graft surgery. Circulation 2008; 118(18):1785-92. 8. Zhang H, Yuan X, Zhang H, Chen S, Zhao Y, Hua K, et al. Efficacy of Long-Term $\beta$-Blocker Therapy for Secondary 
Prevention of Long-Term Outcomes After Coronary Artery Bypass Grafting Surgery. Circulation. 2015; 131(25):2194201.

9. Levitskaya ES, Chesnikova AI, Batiushin MM, Terent'ev V.P. Assessing the impact of renal risk factors on the probability of recurrence of angina pectoris in patients undergoing myocardial revascularization, optimization of drug therapy. Arch Inter Med. 2012;(5):45-50. [In Russian].

10. Ferrario CM, Strawn WB. Role of the renin-angiotensinaldosterone system and proinflammatory mediators in cardiovascular disease. Am J Cardiol. 2006; 98(1): 121-8.

11. Minuzzo L, Santos ES, Timerman A. Association between angiotensin-converting enzyme inhibitors and troponin in acute coronary syndrome. Arq Bras Cardiol. 2014; 103(6): 513-20.

12. Agarwal R. Effects of statins on renal function. Mayo Clin Proc. 2007; 82(11): 1381-90.

13. Wanner C, Krane V, März W, Olschewski M, Mann JF,
Ruf $\mathrm{G}$, et al. Atorvastatin in patients with type 2 diabetes mellitus undergoing hemodialysis. N Engl J Med. 2005; 353(3): 238-48.

14. Fellström BC, Jardine AG, Schmieder RE, Holdaas H, Bannister K, Beutler J, et al. Rosuvastatin and cardiovascular events in patients undergoing hemodialysis. N Engl J Med. 2009; 360(14):1395-407.

15. Baigent C, Landray MJ, Reith C, Emberson J, Wheeler DC, Tomson $\mathrm{C}$, et al. The effects of lowering LDL cholesterol with simvastatin plus ezetimibe in patients with chronic kidney disease (Study of Heart and Renal Protection): a randomised placebo-controlled trial. Lancet. 2011; 377(9784):2181-92.

16. Tan KS, Johnson DW. Managing the cardiovascular complications of chronic kidney disease. Aust Prescr 2008; 31: 154-8.

17. Bakris GL. Slowing nephropathy progression: focus on proteinuria reduction. Clin J Am Soc Nephrol. 2008; 3 Suppl 1:S3-10. 